\title{
Possible Role of Sodium-Calcium Pumps in Tension Development of Vascular Smooth Muscle ${ }^{1,2}$
}

\author{
David F. Bohr, Charles Seidel, and Joan Sobieski \\ Department of Physiology, University of Michigan, \\ Ann Arbor, Michigan 48104
}

Received May 7, 1969

\begin{abstract}
Observations were made on the tension development by helical strips of vascular smooth muscle from rabbit aorta and mesenteric artery. Based on the possibility that the pump which moves calcium into the cell coupled with sodium efflux, recently described for squid axon (1), exists also in vascular smooth muscle, studies were designed to evaluate changes in its activity in this muscle. Sodium competes with calcium for the carrier at the cell surface, hence the pump moves calcium into the cell more rapidly in a sodium-free medium. The strips developed more tension in a sodium-free solution, as if more ionized calcium were being delivered to the cell. Ouabain is known to block the sodium-potassium pump, thereby permitting an increase in sodium concentration inside the cell; this in turn will aceclerate the sodium-calcium pump. Ouabain potentiated the contractile responses of these muscles, suggesting that a higher concentration of ionized calcium is reaching the contractile protein.
\end{abstract}

\section{INTRODUCTION}

Ringer (22) alerted the biological world to the fact that shifts in concentration of specific electrolytes predictably alter the mechanical response of muscle. In the intervening century, studies relating changes in contractility to specific shifts in electrolyte concentration have been popular because of the potent effect of these shifts and because of the interesting individualities of the effects of these alterations on contractility of different types of muscles. Such studies on vascular smooth muscle have received additional impetus from the observation that vascular resistance in the hypertensive patient is manipulable by the amount of ingested sodium. Attempts to organize the accumulating masses of information dealing with electrolytes and vascular smooth muscle contraclion have been made in several recent reviews $(4,13,23)$.

The golden fleece of this quest has been understanding of mechanisms by which shifts in electrolyte concentration are capable of altering the ability of the muscle to develop tension. One small bit of this fleece has been found and is now secure in biological literature: the free calcium ion is the essential final common requirement for all muscle contraction. The amount of tension developed by the contractile protein is a direct function of the concentration of this ion to which it is exposed; the concentration of calcium in the cell which will transform the muscle from one with no active tension to one with maximal active tension is extremely low (12). The quest has now shifted to one of understanding the mechanism by which changes in extracellular and intracellular

${ }^{1}$ Presented as part of the Symposium on Vascular Smooth Muscle held at the Microcirculatory Meetings, April 1969.

${ }^{2}$ These studies were supported by grant number HE-03756 from the National Institutes of Health. 
concentrations of sodium, potassium, calcium, and magnesium alter the concentration of the calcium that plays this important regulatory role in the final common pathway.

This orientation of the quest implies that any effective shift in concentration of an electrolyte produces its effect on muscle contraction only by its ability to alter the concentration of free ionized calcium in the environment of the contractile protein. For completeness it must be recognized that electrolyte shifts might alter other rate-limiting processes for muscle contraction; for instance, the electrolyte shift might influence the level of activity of metabolic pathways leading to the production of the energy source for contraction (ATP). Alternatively, the possibility must be considered that tension development by the contractile protein may be influenced by specific ions other than calcium. Magnesium is certainly required but under physiological conditions its intracellular concentration is probably supraoptimal so that it is not rate-limiting for tension development. Consideration of these mechanisms by which electrolyte shifts might alter tension development, other than through an effect they might have on calcium concentration are necessary only for completeness. There is no good evidence that these alternative rate-limiting mechanisms for tension are influenced by shifts in electrolyte concentration.

Although there is no evidence that physiologically occurring ions other than calcium and magnesium have a specific influence on tension development by contractile protein, tension is critically dependent on total ionic strength regardless of the species of ion (19).

Recognizing that under physiological conditions the determinant and final common pathway for tension development is the concentration of free ionized calcium in the environment of the myofilaments and, noting that shifts in any of the four important physiologically occurring cations (sodium, potassium, magnesium, and calcium) are capable of altering tension development, the question that emerges is: How does a shift in a particular ion concentration either intracellularly or extracellularly alter the concentration of the free ionized calcium at its active site? All answers to this question deal with the influence that the ion has on some property of the cell membrane or sarcoplasmic reticulum.

With the current intrusion of knowledge into the electrophysiological workings of the membrane, the most popular hypothesis accounting for the effect of an electrolyte shift on calcium availability to the contractile protein is that this shift produces a change in membrane excitability. Based on what is known of transmembrane concentration gradients of electrolytes and of specific ion permeabilities, mechanisms are described which would cause a decrease in transmembrane voltage, thereby increasing the excitability of the membrane by bringing it closer to a "critical firing potential". One obvious means by which this could be done is through an increase in extracellular potassium concentration. It has been shown by direct observation that the membrane potential of vascular smooth muscle is an appropriate function of the $[K]_{\mathrm{i}}:[\mathrm{K}]_{0}$ ratio (20). In smooth muscle, to a much greater extent than in cardiac and skeletal muscle, the membrane potential is influenced by the transmembrane concentration gradient of sodium. This is true since membrane permeability to sodium is much higher in the nonstriated muscle (16). For this reason it is to be anticipated that an elevation in the $[\mathrm{Na}]_{0}:[\mathrm{Na}]_{i}$ ratio might significantly decreasc resting membranc potential and therefore increase excitability. Also potent in altering membrane potential is the effect that specific ions have on the permeability of the membrane to ions whose transmembrane gradient is contributing to the 
membrane potential. Of the physiologically occurring ions, calcium is the most effective manipulator of membrane permeability. An increase in the amount of calcium in the membrane brings about stabilization and a decrease in excitability $(3,9)$.

The oscillatory process of membranes is also acutely sensitive to the electrolyte environment. The pacemaker activity of the vascular smooth muscle cell is highly dependent on its ionic environment (5). Changes in frequency of firing that result from shifts in electrolyte concentration are important determinants of the amount of tension developed by some vascular smooth muscle.

This summary of the role of membrane excitability and its influence on tension development is based on the classical electrophysiological concepts related to the generation of an action potential as a prerequisite for tension development. Strong evidence has been outlined recently which argues that vascular smooth muscle has physiologically important nonelectrical activation pathways $(6,24)$. The influence of electrolytes on these nonelectrical pathways for activation and contraction has not been worked out, but may have electrolyte dependencies which are very similar to those for the classical electrical activation pathways.

Membrane excitability, as it has been dealt with in the preceding paragraph, has been based on more or less passive phenomena of diffusion potentials and specific membrane permeability to different ion species. Active energy-requiring processes have been recognized as being involved in several ways in membrane excitation and tension development: (1) Aclive pumps are essential in establishing the transmembrane concentration gradient of ions which is responsible for the diffusion potentials. (2) Active translocation of a single ion from one side of the membrane to the other without a coupled movement of an ion of similar charge in the opposite direction will lead to a charge separation (electrogenic pump) (11). (3) Finally, an energy-requiring process (either an active pump or active binding) is essential for the production of relaxation by lowering of calcium in the environment of the myofilament to a level below which the contractile process is not activated (17).

A recent study by Baker et al. (1) has characterized a type of transmembrane electrolyte pump which may have a much more direct and potent influence on the contractile process than do those considered in the preceding paragraph. These investigators have demonstrated that in the membrane of the squid axon there exists an active transmembrane electrolyte pump which couples sodium efflux to calcium influx. Variables which alter the activity of this pump produce parallel changes in sodium efflux and calcium influx. In the squid axon a sodium-potassium pump which is ouabain sensitive is more potent in producing sodium extrusion than is the sodium-calcium pump. The directions of ionic movement produced by these two pumps are shown in Fig. 4; the sodium-potassium pump above and the sodium-calcium in the middle. A third system which may have a direct influence on the contractile process is a calcium-sodium exchange system in which the extrusion of calcium from the cell is energized by the movement of sodium into the cell down its electrochemical gradient. This is the lowermost pump illustrated in Fig. 4. The goal of the current study was to make observations which would evaluate possible applicability of these three pumps to the control of the contraction of vascular smooth muscle. Of particular interest was the possibility that the calcium influx from the sodium-calcium pump might be directly available to the contractile protein and hence to tension development. 


\section{METHODS}

Mesenteric arteries (400-500 $\mu$ od) and aortas were removed from rabbits weighing $2-3 \mathrm{~kg}$. Helical strips were cut from both vessels, those from the aorta were $10 \mathrm{~mm}$ long and 1.5 wide; those from the mesenteric artery were approximately $5 \mathrm{~mm}$ by $.3 \mathrm{~mm}$. The helical strips were suspended at $37^{\circ}$ and $\mathrm{pH} 7.4$ in physiological salt solution (PSS) of the following composition, expressed in millimoles per liter: $\mathrm{NaCl}, 130.0 ; \mathrm{KCl}, 6.0$; $\mathrm{MgCl}_{2}, 1.2 ; \mathrm{CaCl}_{2}, 1.6$; Tris (hydroxymethy) aminomethane, 5.0 , neutralized to $\mathrm{pH} 7.4$ with $\mathrm{HCl}$; glucose, 5.5; sucrose, $60.0 ; \mathrm{CaNa}_{2}$ EDTA, 0.026. An identical solution was made in which $\mathrm{LiCl}$ was substituted for the $\mathrm{NaCl}$ (sodium-free solution). Prior to use the solutions were equilibrated with oxygen at atmospheric pressure. The strip of mesenteric artery was stretched to a resting tension of approximately $200 \mathrm{mg}$ and the aorta was stretched to $600 \mathrm{mg}$. Strips were allowed to equilibrate in the PSS for at least $2 \mathrm{hr}$ before experimental conditions were introduced. Isometric tension was recorded by means of a Grass FT-03 linear-force transducer in connection with a Grass polygraph recorder. The vascular smooth muscle was stimulated with norepinephrine in concentrations which would give approximately $1 / 2$ maximal tension. Ouabain was made up in an aqueous stock solution $\left(10^{-3} \mathrm{M}\right)$ and added to the bath to give a concentration of $10^{-5} \mathrm{M}$ which in the squid axon has been demonstrated to block the sodium-potassium pump, but have no effect on the sodium-calcium pump (1).

\section{RESULTS}

Sodium-free solution. An essential feature of the sodium-calcium pump described by Baker et al. (1) is that the carrier which picks up calcium from the external environment may also be occupied by sodium. These investigators observed that removal of sodium from the external environment led to a great increase in both calcium influx and sodium efflux, presumably because the external carrier had access to more calcium. If such a system were effective in increasing the calcium available for the contractile process, it would be predicted that the absence of $\mathrm{Na}_{0}$ should potentiate a contractile response. Results in the current experiment are in keeping with the efficacy of such a mechanism. Responses of both mesenteric artery and aortic vascular smooth muscle to norepinephrine were potentiated when sodium was replaced by Li. Results in vascular smooth muscle from these two different sources were qualitatively similar (Figs. 1 and 2).

In the cxpcriments shown in Figs. 1 and 2, the bath was changed from sodium to sodium-free $30 \mathrm{sec}$ before the muscle was stimulated with norepinephrine. From these observations and from studies in which the time requirement for this potentiating effect was determined the potentiating effect seemed to be immediate (within seconds after the external sodium had been replaced with $\mathrm{Li}$ ). This suggests that the sodium-calcium pump immediately facilitates the contractile event produced by norepinephrine and raises the question of whether this contractile event may be dependent in part on a norepinephrine facilitation of this pump.

In the absence of norepinephrine a change from sodium salt solution to a sodium-free solution initiates a contraction of the mesenteric artery within $1.5 \mathrm{~min}$. It would appear that the pump is effective in producing a threshold elevation in ionized calcium within the cell without further stimulation. Contraction develops more slowly in the aorta placed in a sodium-free solution. 
It was further observed that in the presence of the sodium-free bath, relaxation of either type of vascular smooth muscle was greatly delayed or prevented (Fig. 2). This delay would be anticipated if the removal of ionized calcium from the environment of the myofilaments were delayed by a failure of a calcium-sodium exchange system (lowermost pump in Fig. 4) in which the movement of sodium into the cell is necessary to drive calcium out or into a membrane trap. In Fig. 2 it is evident that the substitution of sodium for $\mathrm{Li}$ in the bath initiated a fall in tension within $1.5 \mathrm{~min}$.

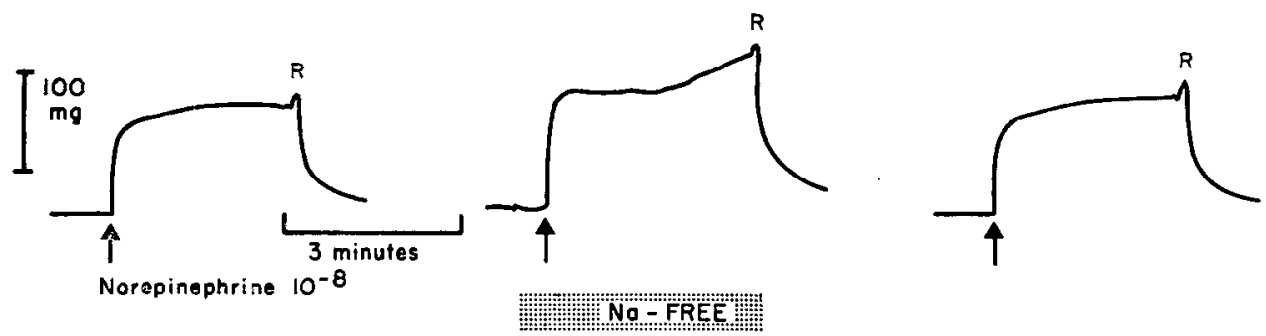

FIG. 1. Mesenteric artery; response to norepinephrine in a sodium-free (Li substituted) bath. The first and third contractions are in a normal sodium environment. Na-free PSS was substituted for normal PSS $30 \mathrm{sec}$ before the muscle was stimulated by norepinephrine in the center tracing. $R=$ bath rinsed.

Ouabain. In the consideration of active pumps involved in transmembrane flux of electrolytes, Baker et al. (1) emphasized that in the squid axon the sodium-potassium pump (uppermost pump in Fig. 4) is probably the most potent. This pump is poisoned by ouabain. When the pump is turned off by this glycoside, sodium accumulates intracellularly. This elevation in intracellular sodium concentration speeds up the pumping action of the sodium-calcium exchange system (middle pump, Fig. 4) leading

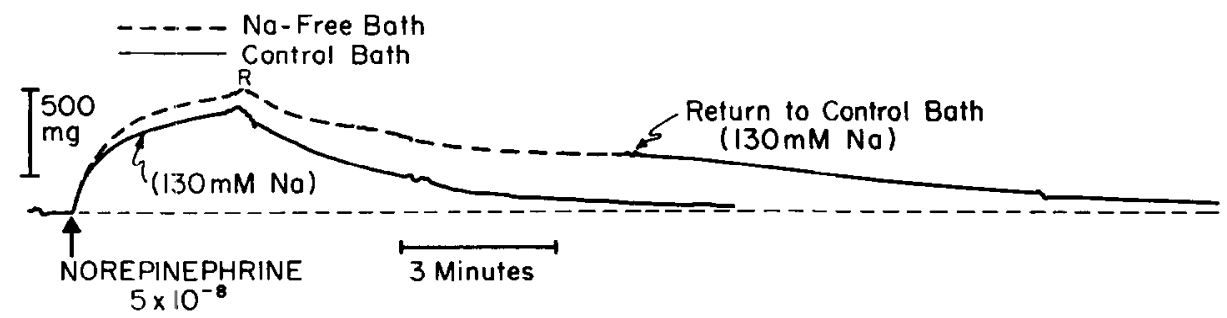

Fig. 2. Aorta; effect of sodium-free solution (Li substituted) on norepinephrine response and on rate of relaxation. The superimposition of the response in the sodium-frec bath cmphasizes the greatcr tension development and lower rate of relaxation displayed by this muscle. $R$ - bath rinsed. When the strip which had responded in a sodium-free bath was placed in control bath, a more abrupt relaxation commenced.

to an increase in calcium influx. If this added calcium is delivered in an ionic form to the environment of the myofilaments a change in the mechanical response would occur. The effects of ouabain on the contractile response and on the baseline tension of smooth muscle from the mesenteric artery are illustrated in Fig. 3. The effects of ouabain on smooth muscle from the aorta are similar, but less pronounced. Introduction of ouabain $30 \mathrm{sec}$ before the second injection of norepinephrine in Fig. 3 caused a rapid potentiation 


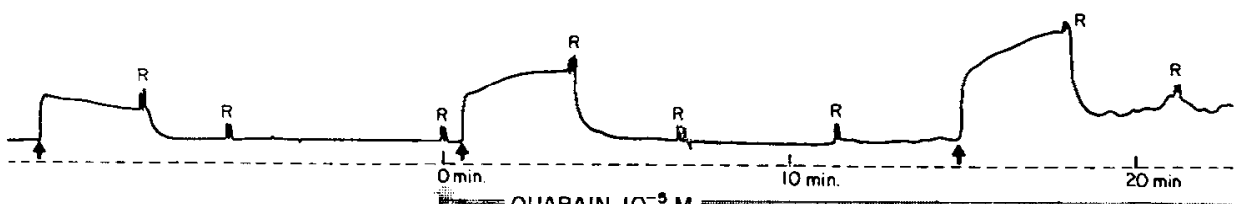

$\uparrow=$ NOREPINEPHRINE $\quad R=$ RINSE

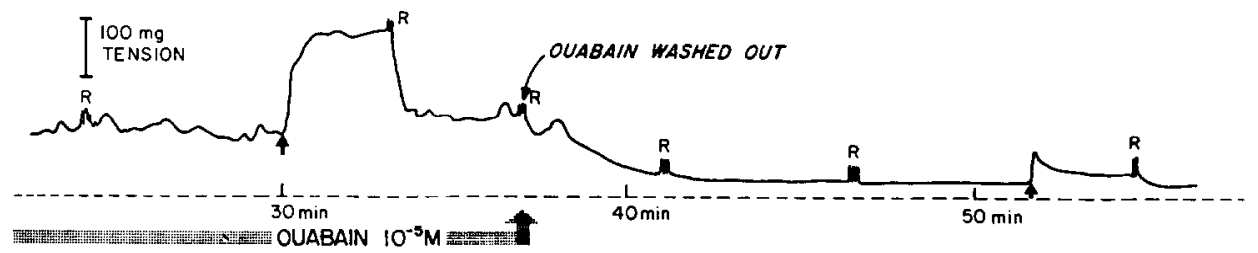

FIG. 3. Mesenteric artery; effect of ouabain on norepinephrine response. The first response in the upper tracing occurred in a normal physiological salt solution. The second, third, and fourth responses occurred in the presence of ouabain $10^{-5} \mathrm{M} . \mathrm{R}=$ bath rinsed. These responses are potentiated by ouabain and baseline tension develops in the unstimulated muscle. This tension is lost when ouabain is washed from the bath.

of the response. There was no change in the fast component of the response to norepinephrine that occurred within the first $5 \mathrm{sec}$, but subsequent tension which in the control response tended to diminish over a 3-min period, underwent a marked increase. This observation is compatible with the possibility that the initial fast component of the response is independent of the sodium-calcium pump, but that the subsequent maintenance of tension reflects calcium being delivered to the myofilaments by the sodiumcalcium pump. Following the initial response in the presence of ouabain, relaxation was
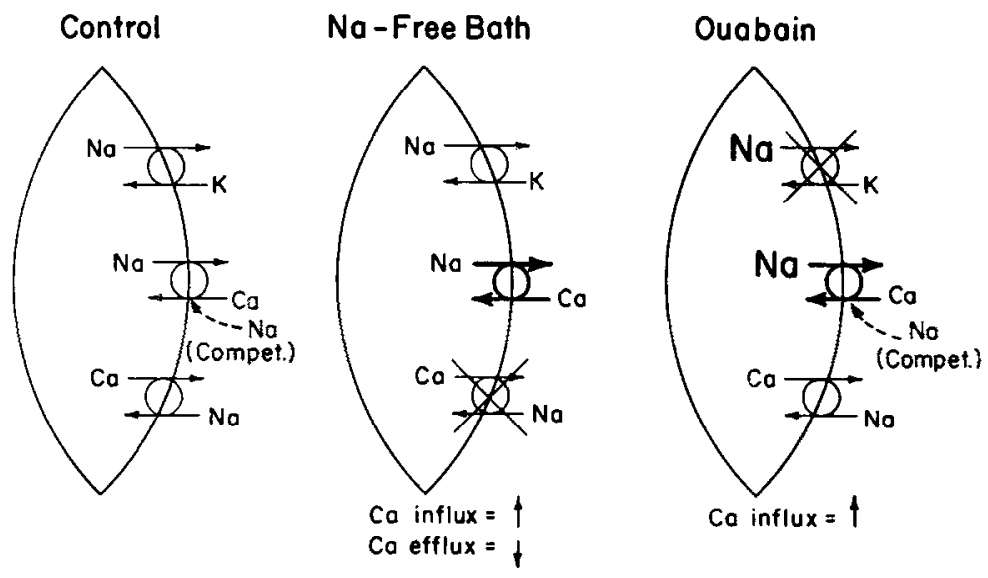

FIG. 4. Schematic model illustrating hypothetical pump systems (or exchange diffusion) responsible for the effects observed in the current study. In each ccll three pumps are illustrated: the uppermost is a ouabain-sensitive pump which exchanges sodium for potassium; the middle pump, a sodium-calcium exchanger, may be accelerated by either removal of external sodium which competes with calcium for a carrier or by increasing the concentration of intracellular sodium by poisoning the ouabain-sensitive sodium-potassium pump; the lowermost exchange system, which may be driven by the transmembrane concentration gradient of sodium, is pictured as being responsible for calcium efflux. 
rapid and complete. However, within $15 \mathrm{~min}$ and prior to the second stimulation with norepinephrine, some spontaneous tone developed. This would be expected if the intracellular sodium concentration had increased to a point where the sodium-calcium pump was effective in developing a threshold concentration of calcium inside the cell. Subsequent responses to norepinephrine were further potentiated, again suggesting that the norepinephrine response may be augmented by a facilitation of this pump. When ouabain was washed from the bath after $35 \mathrm{~min}$ the baseline tension gradually fell to a control level, presumably reflecting a change in intracellular sodium concentration and hence removing the drive of the sodium-calcium pump which had been responsible for elevating the concentration of ionized calcium inside of the cell.

\section{DISCUSSION}

Tension changes that have been observed in the current study are in accord with the possibility that various pumps transport electrolytes across the membrane of the vascular smooth muscle and may play a direct role in altering the concentration of ionized calcium inside of the cell and hence in tension development. The observations can be interpreted largely in terms of what would be expected by alterations of a pump which couples sodium efflux to calcium influx. Such a pump has been described in the squid axon membrane (1). Because sodium and calcium compete for the influx system of this pump, calcium influx is exaggerated in a sodium-free environment. We have observed that such an environment not only potentiates a norepinephrine response, but also is capable of producing a gradual increase in tension without external stimulus. Further support for the role that this pump may play in the development of tension is seen when the sodium-calcium pump is driven from the inside by an increase in intracellular sodium concentration. This presumably can be achieved by ouabain poisoning of the sodium extrusion system (sodium-potassium pump). Here again evidence for an increase in available ionized calcium (tension development) occurred in a time pattern that suggests that it is responding to an increase in intracellular sodium concentration.

The current interpretation based on the contribution of active pumping to the tension development in smooth muscle is supported by observations which demonstrate that these pumps in this tissue are capable of rapid movement of sodium and calcium $(7,14)$.

Observations in the present study suggesting an important role of the active pumps in the contractile process of the vascular smooth muscle, receive strong support from the literature. Over a decade ago we noted that the response of the rabbit aorta was potentiated by a reduction in sodium concentration and depressed by an elevation in concentration of this ion (2). Most directly supportive of the role of the sodium-calcium pump in tension development are the studies by Briggs and Melvin, who observed that when rabbit aortic strips were exposed to a low sodium concentration, calcium influx increased by $225 \%$ and contracture developed (7).

Also supportive of the competition that exists between sodium and calcium for the influx pathway in the sodium-calcium pump of smooth muscle is the observation by Goodford (15) that the concentration of calcium in the taenia coli is increased when sodium is removed from the bathing medium. That this system is of significance in various tissues is suggested by Niedergerke's (21) observation that extra calcium influx 
per beat in the frog ventricle increased when sodium concentration of the salt solution was reduced to $50 \%$. A similar increase in influx could be achieved by doubling the calcium concentration while holding sodium concentration constant. Both environmental changes produced a positive inotropic effect which appeared to reflect the observed increases in calcium influx. He interprets this observation as indicating that calcium competes with sodium for a system that moves the calcium into the cell.

Evidence developed from the use of cardiac glycosides extensively supports the importance of the sodium-calcium pump in the determination of tension development. Leonard (18) observed that strophanthidine both potentiated the response of helical strips from the rabbit carotid artery (electrical stimulation) and caused a contracture. Although he attributed these effects to a different mechanism, they are entirely in accord with our current observations. Brinley (8) observed that strophanthidine reduced sodium efflux by $80-90 \%$ and caused contracture of the barnacle muscle. In this giant muscle cell he was able to control intracellular sodium concentration by microinjection or external loading. With this technique he found that there was a strophanthidineinsensitive system capable of producing sodium efflux and that activity of this system was greatly increased as the concentration of sodium inside of the cell was increased.

In his recent review of factors governing movement of ions, Caldwell (10) cites specific evidence supporting the possibility that ouabain causes an increase in calcium influx associated with inhibition of the active movement of potassium and sodium. $\mathrm{He}$ points out that an increase in intracellular sodium would activate a sodium-calcium pump thereby increasing calcium entry.

Clearly, the current results can constitute no more than indirect suggestions that ionic exchange systems which have been identified in the squid axon may have relevance in the contractile process of vascular smooth muscle. There is broad support, however, for a direct role of a sodium-calcium pump in determining the concentration of intracellular ionized calcium and hence in establishing the level of contraction of vascular smooth muscle. The effect that shifts in electrolyte concentrations may have on the activity of this pump must therefore be reckoned with, in an understanding of the mechanism by which these shifts produce their effects on contraction of this muscle.

\section{REFERENCES}

1. Baker, P. F., Blaustein, M. P., Hodgkin, A. L., and Steinhardt, R. A. (1969). The influence of calcium on sodium efflux in squid axons. J. Physiol. 200, 431-458.

2. Bohr, D. F., Brodie, D. C., AND CheU, D. H. (1958). Effect of electrolytes on arterial muscle contraction. Circulation 17, 746-749.

3. BoHR, D. F. (1963). Vascular smooth muscle: Dual effect of calcium. Science 139, 597-599.

4. BoHR, D. F. (1964). Electrolytes and smooth muscle contraction. Pharmacol. Rev. 16, 85-111.

5. BoHR, D. F. Individuality in smooth muscle control. In "Proceedings of a Workshop on Ureteral Rcflux in Children," Nov. 11-12, 1966 (James F. Glenn, ed.), Nat'l Acad. Sci. Nat'l Research Council Publ., pp. 57-66.

6. BoHR, D. F., AND UCHIDA, E. (1969). Activation of vascular smooth muscle. In "The Pulmonary Circulation and Interstitial Space," (A. Fishman and H. Hecht, eds.), Univ. Chicago Press, Chicago, Illinois. Chap. 10, pp. 133-145.

7. BRIGGS, A. H., AND MELVIN, S. (1961). Ion movement in isolated rabbit aortic strips. Am.J.Physiol. 201, 365-368.

8. BRINLEY, F. J. (1968). Sodium and potassium fluxes in isolated barnacle muscle fibers. J. Gen. Physiol. 51, 445-477. 
9. Burnstock, G., Holman, M. E., And Prosser, C. L. (1963). Electrophysiology of smooth muscle. Physiol. Rev. 43, 482-527.

10. CALDWELL, P. C. (1968). Factors governing movement and distribution of inorganic ions in nerve and muscle. Physiol. Rev. 48, 1-64.

11. Daniel, E. E. (1963). K movements in rat uterus studied in vitro II. Effects of metabolic inhibitors, ouabain and altered K concentrations. Can. J. Physiol. Biol. 41, 2085-2105.

12. Filo, R. S., AND BoHR, D. F. (1965). Glycerinated skeletal and smooth muscle: Calcium and magnesium dependence. Science 147, 1581-1583.

13. Friedman, S. M., and Friedman, C. L. (1963). Effects of ions on vascular smooth muscle. In "Handbook of Physiology: Circulation II," (W. F. Hamilton and P. Dow, eds.), pp. 1135-1166.

14. Goodford, P. J., ANd Hermansen, K. (1961). Na and K movements in the unstriated muscle of the guinea-pig taenia coli. J. Physiol. 158, 426-448.

15. Goodford, P. J. (1967). The calcium content of the smooth muscle of the guinea-pig taenia coli. J. Physiol. 192, 145-157.

16. HaGemeiJer, F., Rorive, G., AND SChOfFEniels, E. (1965). Exchange of ${ }^{24} \mathrm{Na}$ and ${ }^{42} \mathrm{~K}$ in rat aortic smooth muscle fibers. Life Sci. 4, 2141-2149.

17. Hasselbach, W. (1964). Relaxation and the sarcotubular calcium pump. Federation Proc. 23, 909-912.

18. LEONARD, E. (1957). Alterations of contractile response of artery strip by a potassium-free solution, cardiac glycosides and changes in stimulation frequency. Am. J. Physiol. 189, 185-190.

19. Murphy, R. A., AND Koss, P. G. (1968). Hydrogen ion buffers and enzymatic activity: Myosin B adenosinetriphosphatase. Arch. Biochem. Biophys. 128:236-242.

20. NAKAJIMA, A., AND HORN, L. (1967). Electrical activity of single vascular smooth muscle fibers. Am. J. Physiol. 213, 25-30.

21. Niedergerke, R. (1963). Movements of $\mathrm{Ca}$ in frog heart ventricles at rest and during contractures. J. Physiol. 167, 515-550.

22. RINGER, S. (1883). A further contribution regarding the influence of the different constituents of blood on the contraction of the heart. J. Physiol. 4, 29-42.

23. SomLyo, A. P., AND SomLyo, A. V. (1968). Vascular smooth muscle. I. Normal structure, pathology, biochemistry, and biophysics. Pharmacol. Revs. 20, 197-271.

24. Somlyo, A. V., AND Somlyo, A. P. (1968). Electromechanical and pharmacomechanical coupling in vascular smooth muscle. J. Pharmacol. Exptl. Therap. 159, 129-145. 